\title{
Comparative Study of Magnetic Resonance Imaging, Computed Tomograpy and Histology in the Assessment of Liver Iron Overload
}

\author{
Masaru Harada, Kenji Hirai, Shotaro Sakisaka, Takato Ueno, Hirohiko Abe and Kyuichi TaniKawa
}

\begin{abstract}
Magnetic resonance imaging, computed tomograpy, and liver biopsy findings were compared in ten patients with serum ferritin levels over $500 \mathrm{ng} / \mathrm{ml}$. The liver was observed as a lowintensity area on magnetic resonance imaging in all four patients with serum ferritin levels above $2,000 \mathrm{ng} / \mathrm{ml}$, while no abnormalities were detected in four of the six patients with a serum ferritin level below $2,000 \mathrm{ng} / \mathrm{ml}$. Computed tomography revealed the liver to be a high density area in five of the seven patients tested. However, it demonstrated no abnormality in a patient with steatosis despite a high serum ferritin concentration. Liver biopsy demonstrated iron deposits in nine of the ten patients. These findings indicate that liver biopsy remains the most accurate mean of detection of liver iron overload. Both magnetic resonance imaging and computed tomography could be used to be monitor the progress of a patient with liver iron overload treated by phlebotomy.
\end{abstract}

(Internal Medicine 31: 180-184, 1992)

Key words: hemochromatosis, ferritin

\section{Introduction}

The liver is the main organ for the storage of iron; $60 \%$ of the body's iron stores are located in the liver (1). Excretion of iron from the skin, gastrointestinal tract, and genitourinary tract only totals about $1 \mathrm{mg} /$ day (2), and the biliary excretion of ferritin is also limited (3). Therefore, iron accumulation can readily occur in various organs in a number of pathological states, resulting in diabetes mellitus, hypopituitarism, hypothyroidism, adrenal insufficiency, heart failure, arthropathy, skin pigmentation, and liver cirrhosis (1). Accordingly, the assessment of an accumulation of iron in the liver is necessary for diagnosis and adequate therapeutic management of liver iron overload. Histological study of the liver remains the most accurate means of assessing liver iron overload (4), however, liver biopsy is associated with certain unavoidable risks. Biochemical parameters such as the serum iron level, transferrin saturation, and serum ferritin level are of great value for preliminary screening, and the measurement of liver attenuation by computed tomography (CT) is now possible for the detection of liver iron overload.

Iron as a ferric ion does not produce any signal on magnetic resonance imaging (MRI), however, it influences relaxation times as a paramagnetic ion (5). Recently some investigators have reported that MRI is of great value in the diagnosis of iron overload (6-9).

The purpose of the present study was to evaluate the clinical significance of MRI in the assessment of liver iron overload, in comparison with CT and liver biopsy.

\section{Patients and Methods}

At the Second Department of Medicine, Kurume University School of Medicine, MRI and liver biopsy were performed in ten patients with serum ferritin levels more than $500 \mathrm{ng} / \mathrm{ml}$. Patients complicated with malignant neoplasm or inflammation were excluded. CT was also performed in seven of the ten patients. The serum iron concentration and unsaturated serum iron binding capacity (UIBC) were also determined. Normal values were as follows: serum iron concentration, 80 to $170 \mu \mathrm{g} / \mathrm{dl}$; serum UIBC, 180 to $313 \mu \mathrm{g} / \mathrm{dl}$; serum ferritin

From The Second Department of Medicine, Kurume University School of Medicine, Kurume

Received for publication March 11, 1991; Accepted for publication July 10, 1991

Reprint requests should be addressed to Masaru Harada, MD, The Second Department of Medicine, Kurume University School of Medicine, Asahi-machi 67, Kurume 830, Japan 
MRI of Iron Overload in the Liver

Table 1. Clinical and Laboratory Data of Patients

\begin{tabular}{llcccc}
$\begin{array}{l}\text { Case } \\
\text { number }\end{array}$ & Sex & Age & $\begin{array}{c}\text { Fe } \\
(80-170 \mu \mathrm{g} / \mathrm{dl})\end{array}$ & $\begin{array}{c}\text { UIBC } \\
(180-313 \mu \mathrm{g} / \mathrm{d} \mathrm{l})\end{array}$ & $\begin{array}{c}\text { Ferritin } \\
(8-100 \mathrm{ng} / \mathrm{dl})\end{array}$ \\
\hline 1 & M & 55 & 235 & 25 & 6,000 \\
$2^{*}$ & $\mathrm{~F}$ & 55 & 300 & 31 & 4,000 \\
3 & $\mathrm{M}$ & 55 & 158 & 30 & 2,300 \\
4 & $\mathrm{M}$ & 61 & 265 & 38 & 2,210 \\
5 & $\mathrm{M}$ & 62 & 328 & 25 & 1,950 \\
6 & $\mathrm{~F}$ & 69 & 248 & 33 & 1,510 \\
7 & $\mathrm{M}$ & 49 & 255 & 29 & 1,190 \\
8 & $\mathrm{M}$ & 66 & 213 & 31 & 1,190 \\
9 & $\mathrm{M}$ & 66 & 120 & 67 & 1,026 \\
10 & $\mathrm{M}$ & 44 & 196 & 150 & 720 \\
\hline
\end{tabular}

* patient treated by phlebotomy

concentration, 8 to $100 \mathrm{ng} / \mathrm{ml}$. There were eight men and two women with an age range of 44 to 69 year (Table 1).

MRI was performed with a SMT-50 (Shimadzu, Kyoto, Japan) operating at 0.5 Tesla. The pulse sequence was spin echo (SE), with repetition times (TR) of 1,500 to $2,000 \mathrm{~ms}$ and echo times (TE) of 80 to $100 \mathrm{~ms}$ for T2weighted images. The intensity of the liver was compared with the intensity of the flank muscles, and was graded from low to high intensity. Plain CT was peformed with a TCT-60T (Toshiba, Tokyo, Japan) or a GE-CT (General Electric, Milwaukee, U.S.A.), and liver attenuation was measured in the right lobe before contrast enhancement to avoid the biliary and vascular structures. The range of normal liver attenuation was from 52 to 68 Hounsfield units (H.U.). Liver biopsy was performed in all patients and the time interval between MRI and liver biopsy ranged from 1 to 2 months. The obtained liver tissues were processed by hematoxylin-eosin staining for histological diagnosis and prussian blue staining for the determination of iron. The degree of iron accumulation was graded from - to $++(-=$ negative, $+=$ weakly positive, and $++=$ strongly positive). Phlebotomy ( $200 \mathrm{ml} / \mathrm{wk}$, for 18 months) was performed in one patient (case 2), and the findings noted by MRI, CT, and his- tological examination were compared before and after phlebotomy.

\section{Results}

Table 1 summarizes the clinical and laboratory data, and Table 2 summarizes the MRI (T2-weighed images), plain CT, and histological findings. The liver intensity was decreased in six of the ten patients on MRI. Attenuation values for the liver were elevated in five of the seven patients evaluated by CT. Histological study demonstrated iron accumulation in the liver in nine of the ten patients. The liver was demonstrated as a low intensity area on MRI in all four patients with a serum ferritin concentration of over $2,000 \mathrm{ng} / \mathrm{ml}$ (cases 1-4). CT revealed the liver to be a high-density region in two of the three patients with a serum ferritin concentration over $2,000 \mathrm{ng} / \mathrm{ml}$. Reaction products for iron were histologically found in the liver of these patients. In four of the six patients with serum ferritin levels under $2,000 \mathrm{ng} / \mathrm{ml}$ (cases 5-10), MRI showed the liver as an iso-intense structure, while CT showed elevated attenuation values in three of these four patients evaluated by CT. In the histological study, reaction products were

Table 2. Results of MRI, CT Scan and Histological Study

\begin{tabular}{lccccc}
\hline $\begin{array}{l}\text { Case } \\
\text { number }\end{array}$ & $\begin{array}{c}\text { Intensity } \\
\text { of MRI }\end{array}$ & $\begin{array}{c}\text { CT } \\
(\text { H.U. })\end{array}$ & $\begin{array}{c}\text { Liver biopsy } \\
\text { iron deposit }\end{array}$ & $\begin{array}{c}\text { Histological } \\
\text { diagnosis }\end{array}$ & Steatosis \\
\hline 1 & low & 49 & ++ & LC & + \\
$2^{*}$ & low & 91 & + & HF & - \\
3 & low & 70 & + & LC & - \\
4 & low & NE & ++ & CPH & - \\
5 & iso & NE & - & CAH & + \\
6 & low & 69 & ++ & CAH & + \\
7 & iso & 49 & + & LC & - \\
8 & iso & 70 & ++ & LC & - \\
9 & low & 70 & + & HF & - \\
10 & iso & NE & ++ & ND & - \\
\hline
\end{tabular}

CAH: chronic active hepatitis, CPH: chronic persistant hepatitis, HF: hepatic fibrosis, NE: not examined, ND: not diagnosed, LC: liver cirrhosis

* patient treated by phlebotomy 


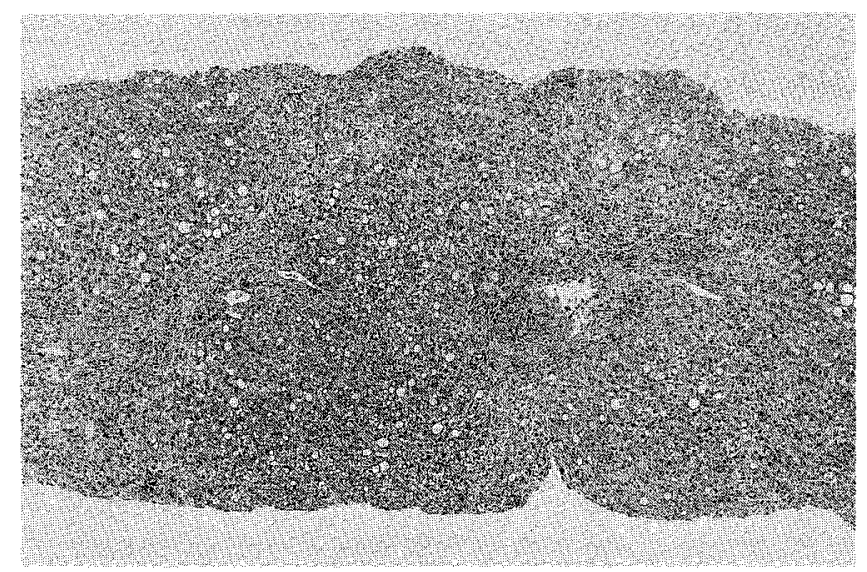

Fig. 1. Light microscopic study of the liver (case 1) with hematoxylin-eosin staining showing mild steatosis $(\times 30)$.

observed in five of these six patients.

Despite a high serum ferritin concentration $(6,300 \mathrm{ng} /$ $\mathrm{ml}$ ) and iron deposition in the liver, the attenuation value was low $(49 \mathrm{H} . \mathrm{U}$.) on CT in case 1 . In this patient, fatty infiltration was noted by histological study of the liver (Fig. 1).

\section{A Representative Case}

A 53-year-old housewife (case 2) was referred to our hospital because of abnormal liver function tests. The patient had been administered ferrous sulfate because of anemia for about a year at another hospital. The serum glutamate pyruvate transaminase (GPT) level was $119 \mathrm{KU}$, the serum iron concentration was $300 \mu \mathrm{g} / \mathrm{dl}$, and the ferritin concentration was $4,000 \mathrm{ng} / \mathrm{dl}$ (Fig. 2). Numerous reaction products were observed in the liver biopsy specimen with prussian blue staining (Fig. 3a). MRI revealed the liver to be a very low signal intensity area (Fig. 4a), and liver attenuation on CT was 91 H.U. (Fig. 5a). Therefore, secondary hemochromatosis was diagnosed and phlebotomy was performed at a rate of

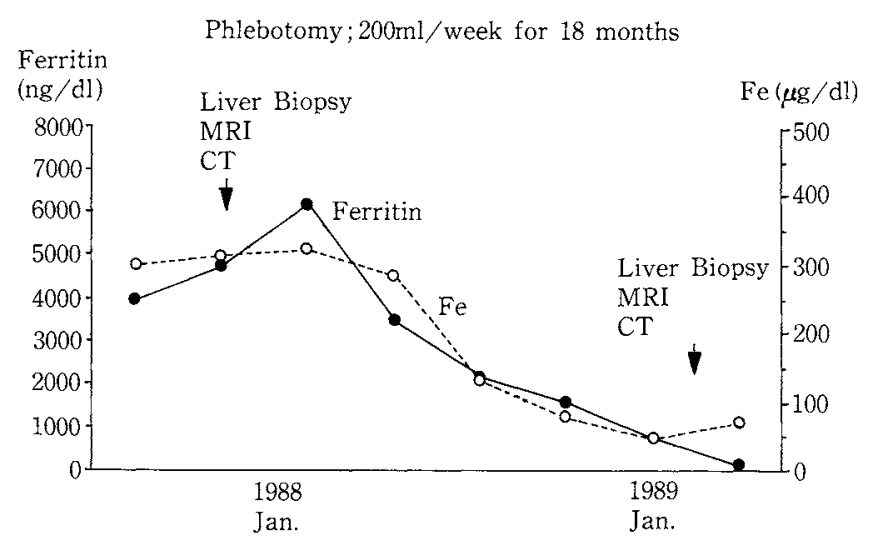

Fig. 2. Clinical course of case 2, who had secondary hemochromatosis treated by phlebotomy. CT, computed tomograpy; MRI, magnetic resonance imaging.

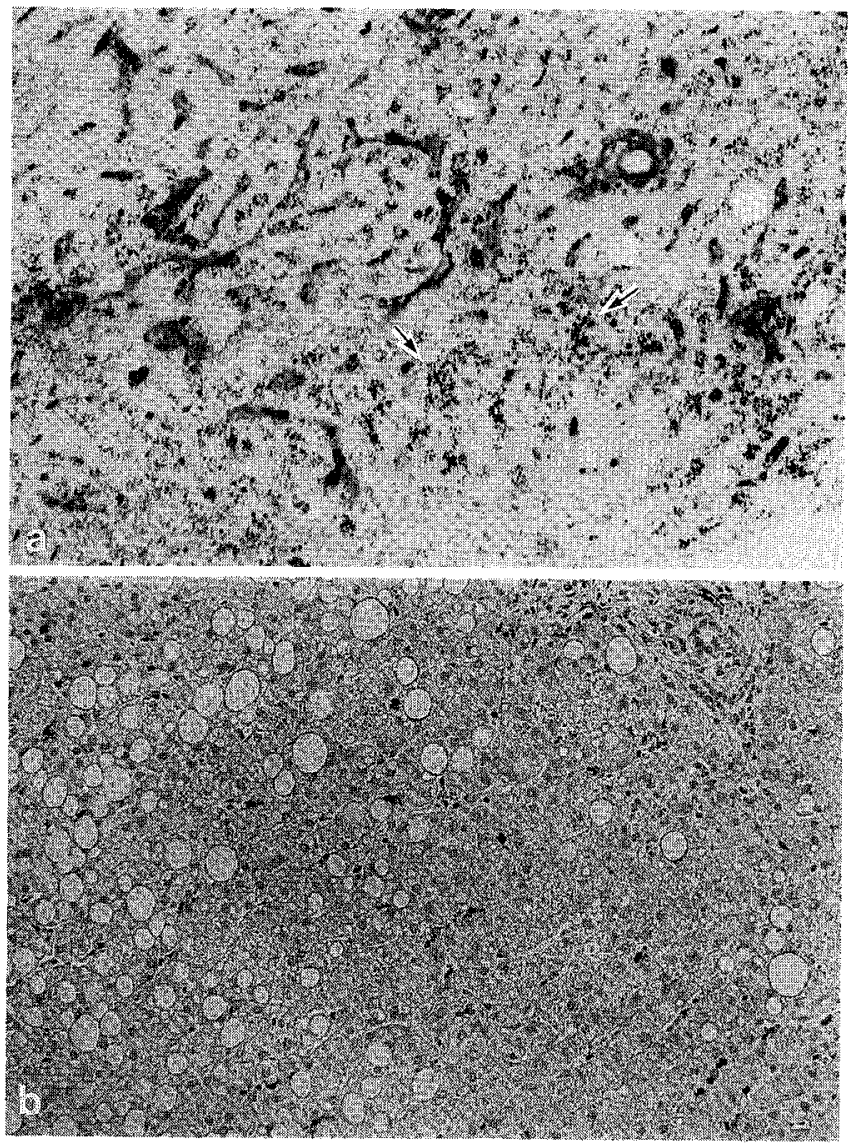

Fig. 3. Light microscopic study of the liver (case 2) with prussian blue staining, before (a) and after (b) phlebotomy. Before phlebotomy many reaction products (arrows) are seen in the liver, after phlebotomy, few reaction products are observable $(\times 150)$.

$200 \mathrm{ml}$ per week for 18 months (total volume of blood extracted: $14,400 \mathrm{ml}$; total iron extracted: $5,760 \mathrm{mg}$ ). After treatment by phlebotomy, the serum GPT was improved to a normal range, and the iron and ferritin concentrations were decreased to $71 \mu \mathrm{g} / \mathrm{dl}$ and $109 \mathrm{ng} / \mathrm{dl}$, respectively. Only a few reaction products were observed in the liver on repeat biopsy (Fig. 3b), the hepatic signal intensity on MRI increased (Fig. 4b), and the liver attenuation on CT decreased to 58 H.U. (Fig. 5b).

\section{Discussion}

MRI is based upon the differences in hydrogen relaxation times. Tissue iron is largely bound to ferritin and hemosiderin as a ferric ion and influences MRI signal intensities as a paramagnetic ion (5). Recently, some investigators have reported that MRI is a very sensitive method for determining iron overload in the liver (6-8). Iron overload in the liver results in a marked alteration of the relaxation time on MRI, and MRI intensity has been correlated with the tissue iron concentration. A concentration of ferric salts of greater than $20 \mathrm{mmol}$ yields a lower intensity signal and a concentration of less 

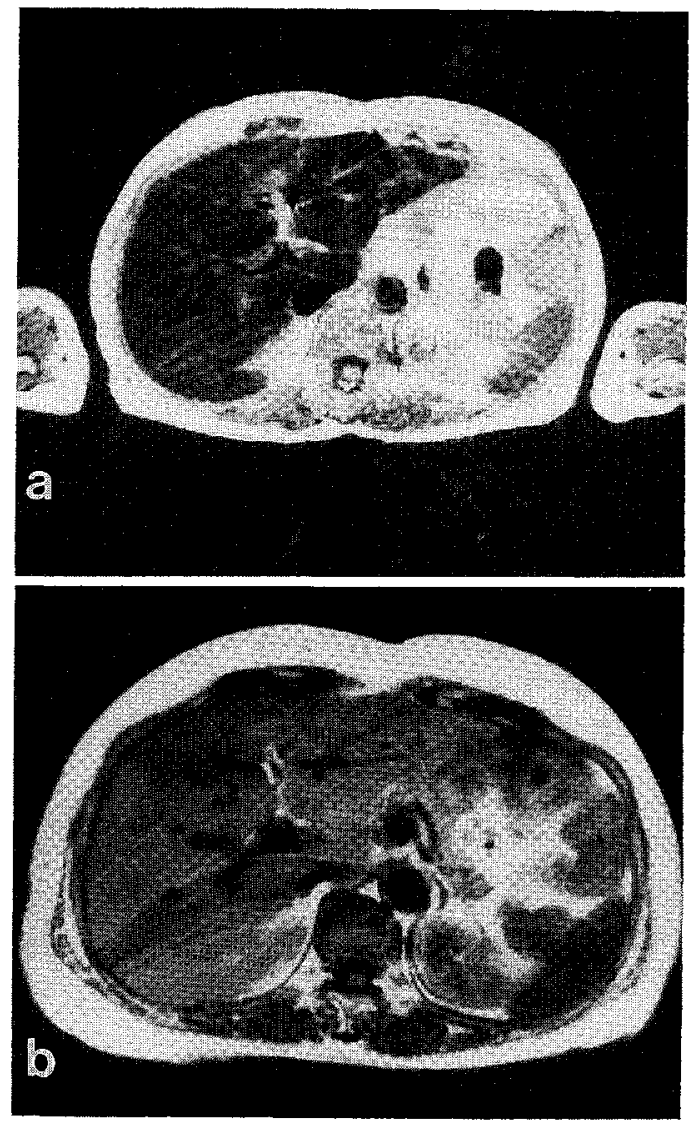

Fig. 4. Magnetic resonance imaging of the liver before and after phlebotomy. Before phlebotomy, the signal intensity of the liver is noted to be very low (a), and after phlebotomy the signal intensity is increase (b).

than $15 \mathrm{mmol}$ yields a higher intensity signal than that of water (7). Iron markedly decreases the signal intensity in T2-weighted images and moderately decreases in T1weighted images (8). Moreover, an increase in liver attenuation on plain CT due to iron overload has been well documented (10). Therefore, we evaluated the differences in efficacy in the assessment of liver iron overload, between MRI (T2-weighed images), plain CT and liver histological study.

Because the serum ferritin level closely reflects body iron stores (2), we selected patients with a serum ferritin level over $500 \mathrm{ng} / \mathrm{ml}$. In all four patients with serum ferritin levels over 2,000 ng/ml (cases 1-4), the liver histological study demonstrated iron accumulation. In these patients, the hepatic signal intensity on MRI was always low. Thus, MRI was useful for the assessment of liver iron overload as well as histological study. However, liver attenuation was not elevated on CT in one of these patients (49 H.U.). In this case, iron deposition in hepatocytes was associated with fatty infiltration of the liver. The reduction in the sensitivity of CT seemed to be related to the presence of fatty liver, as has previously been reported (4). Thus, CT appears not to be useful for determining liver iron overload in the presence of fatty
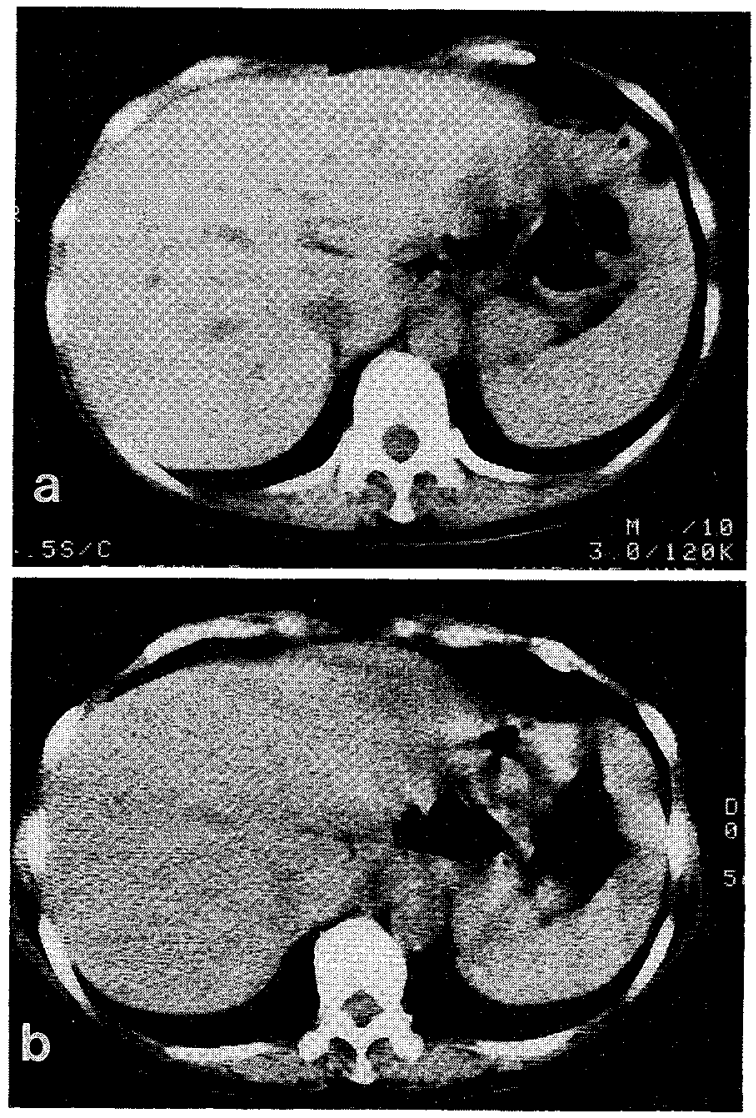

Fig. 5. Computed tomographs of the liver before and after phlebotomy. Before phlebotomy, the density of the liver is high and the attenuation value is 80 H.U. (a), while after phlebotomy the attenuation value is decreased to 58 H.U. (b).

liver. Guyader et al (4) also reported that $40 \%$ of the false negatives on CT could be attributed to an associated steatosis. In another patient (case 6) iron accumulation was also associated with steatosis, however, CT still indicated the liver to be a high density region. This was probably due to a lesser degree of fatty infiltration.

In patients with serum ferritin levels under $2,000 \mathrm{ng} /$ $\mathrm{ml}$, liver histological study demonstrated iron accumulation in five of the six patients. However, MRI did not always reveal the liver as a low signal intensity area. Among these six patients (cases 5-10), the MRI intensity was decreased in two patients, and $\mathrm{CT}$ attenuation values were elevated in three of the four patients scanned. Since cirrhosis alone does not markedly alter the MRI characteristics of the liver $(11,12)$, the present findings were probably due to the limited sensitivity of MRI for mild hepatic iron accumulation. These results indicate that, the histological study is still the most reliable examination for the detection of liver iron overload, and MRI cannot predict liver iron overload in patients with a serum ferritin level under $2,000 \mathrm{ng} / \mathrm{ml}$, as their liver is considered to contain only a moderate amount of iron.

In one case, the disappearance of iron from hepato- 


\section{Harada et al}

cytes after phlebotomy was noted by histological study, and the efficacy of treatment was also evaluated by both MRI and CT. Both MRI and CT demonstrated the improvement after treatment, as did the histological examination.

In conclusion, MRI was useful in determing liver iron overload when the serum ferritin level was over $2,000 \mathrm{ng} / \mathrm{ml}$. CT was also of great value for assessing liver iron overload, however, it has limited clinical value in cases with steatosis. Furthermore, histological study of the liver appears to still be the most accurate examination for the early detection of iron overload in the liver, and both MRI and CT seem useful for evaluating therapeutic effect of phlebotomy.

\section{References}

1) Sherlock S. Iron overload states. in: Disease of the Liver and Biliary System, eighth edition, Sherlock S, Ed. Blackwell Scientific Publications, Boston, Melbourne, 1989, p. 449.

2) Powell LW, Halliday JW. Iron, ferritin, and the liver. in: Progress in Liver Disease, volume 7, Popper $\mathrm{H}$ and Schaffner F, Eds. Grune and Stratton, New York, 1982, p. 599.

3) Hulterantz R, Angelin B, Rasmussen EB, Ewerth S, Einarsson K. Biliary excretion of iron and ferritin in idiopathic hemochro- matosis. Gastroenterology 96: 1539, 1989.

4) Guyader D, Gandon Y, Deugnier Y, et al. Evaluation of computed tomography in the assessment of liver iron overload. Gastroenterology 97: 737, 1989.

5) Hernandez RJ, Sarnaik SA, Lande I, et al. MR evaluation of liver iron overload. I Comput Assist Tomogr 12: 91, 1988.

6) Stark DD, Bass NM, Moss AA, et al. Nuclear magnetic resonance imaging of experimentally induced liver disease. Radiology 148: $743,1983$.

7) Brasch RC, Wesbey G, Gooding CA, Koerper M. Magnetic resonance imaging of transfusional hemosiderosis in children with thalassemia major. Radiology 150: 767, 1984.

8) Stark DD, Moseley ME, Bacon BR, et al. Magnetic resonance imaging and spectroscopy of hepatic iron overload. Radiology 154: 137, 1985.

9) Yoshida H, Mano I, Asai S, Yashiro N, Itai Y, Iio M. Magnetic resonance imaging of iron storage disease. Jpn $\mathbf{J}$ Clin Radiol 30: $1099,1985$.

10) Howard JM, Ghent CN, Carey LS, Flanagan PR, Valberg LS. Diagnostic efficacy of hepatic computed tomography in the detection of body iron overload. Gastroenterology 84: 209, 1983.

11) Stark DD, Henry IG, Albert AM, Bass NM. Chronic liver disease: Evaluation by magnetic resonance. Radiology 150: $149,1984$.

12) Glodberg HI, Moss AA, Mckerrow J, Engelstad B, Brito A. Hepatic chirrhosis: Magnetic resonance imaging. Radiology 153: 737, 1984. 